\title{
Clinical diagnoses among individuals with primary age-related tauopathy versus Alzheimer's neuropathology
}

\author{
Merilee Teylan $^{1} \cdot$ Lilah M. Besser ${ }^{2} \cdot$ John F. Crary $^{3} \cdot$ Charles Mock$^{1} \cdot$ Kathryn Gauthreaux ${ }^{1} \cdot$ Nicole M. Thomas ${ }^{1} \cdot$ \\ Yen-Chi Chen $\mathbb{D}^{1} \cdot$ Walter A. Kukull ${ }^{1}$
}

Received: 14 September 2018 / Revised: 5 November 2018 / Accepted: 19 November 2018 / Published online: 1 February 2019

(c) United States \& Canadian Academy of Pathology 2019

\begin{abstract}
Primary age-related tauopathy (PART) is increasingly recognized as a pathologic entity distinct from Alzheimer's disease (AD). Given that the diagnosis of PART is an autopsy diagnosis, it is unclear how PART is perceived in clinical practice. Thus, we investigated the presumptive primary and contributing diagnoses in individuals who had cognitive impairment while alive and who met neuropathologic criteria for PART at autopsy. We also compared these clinical diagnoses for people with PART to those with AD neuropathology (ADNP). We used data on 1354 participants from the National Alzheimer's Coordinating Center, restricting to those with no neuritic plaques (PART) or moderate/frequent neuritic plaques (ADNP); clinical visit within two years of autopsy; and mild cognitive impairment (MCI) or dementia at last visit. To assess if PART participants were less likely to receive a clinical diagnosis of $\mathrm{AD}$ at their last visit prior to autopsy, we used logistic regression, controlling for age, sex, education, and APOE $\varepsilon 4$ status. There were 161 PART individuals $(n=49 \mathrm{MCI} ; n=$ 112 dementia) and 1193 individuals with $\operatorname{ADNP}(n=75 \mathrm{MCI} ; n=1118$ dementia). Primary clinical diagnosis of AD was more common in those with ADNP (MCI: 69\%; demented: 86\%) than PART (MCI: 57\%; demented: 52\%). In the adjusted analysis, primary and contributing clinical diagnoses of AD remained less likely in PART vs. ADNP participants with dementia (OR: $0.22,95 \%$ CI: 0.13-0.38). This study suggests that clinicians recognize a distinction in the clinical presentation between PART and ADNP, diagnosing AD less frequently in those with PART. Nonetheless, clinical AD was diagnosed greater than $50 \%$ of the time in PART participants with MCI or dementia. Ante-mortem criteria for diagnosis of PART need to be established, as PART is a neuropathological entity that is distinct from AD and has its own clinical and cognitive outcomes.
\end{abstract}

\section{Introduction}

Increasing attention is being given to the neuropathological entity of primary age-related tauopathy (PART). This has been recognized as a neuropathologically overlapping yet separate category from Alzheimer's disease (AD). Both

Merilee Teylan

mateylan@uw.edu

1 National Alzheimer's Coordinating Center, Department of Epidemiology, University of Washington, Seattle, WA, USA

2 Institute for Healthy Aging and Lifespan Studies, School of Urban and Regional Planning, Florida Atlantic University, Boca Raton, FL, USA

3 Departments of Pathology and Neuroscience, Friedman Brain Institute, Ronald M. Loeb Center for Alzheimer's Disease, Icahn School of Medicine at Mount Sinai, New York, NY, USA have similar features of neuronal tau deposits and eventual temporal lobe neurodegeneration. However, PART arises in the absence of the amyloid deposits that currently typify AD. The two conditions also have different clinical manifestations, with PART arising at an older age and being less likely to lead to more severe cognitive dysfunction [1-4].

Thus far, most studies of PART have focused on autopsy findings, with only a handful of studies investigating the clinical diagnoses given to individuals who are later determined to have PART at autopsy. Several older studies, which predate the establishment of PART as a separate diagnostic category, did report on symptoms and diagnoses of people with senile dementia of the tangle type or with neurofibrillary tangle predominant form of senile dementia, both of which are older terms encompassing cases on the severe end of cases now considered on the PART continuum $[2,5,6]$. While amnestic symptoms were considered dominant in these small studies, psychiatric (e.g., psychotic) 
symptoms were also noted [5]. One of these earlier studies reported on 11 cases, all of whom had been diagnosed with possible or probable AD during life [6-8].

More recently, biomarker studies have increased the recognition of amyloid negative yet neurodegeneration positive persons. This has been termed suspected non-Alzheimer disease pathophysiology (SNAP) and appears to overlap with PART. Similar to PART, persons with SNAP test negative for amyloid deposits in the brain, but have other markers of neurodegeneration; although SNAP is based on ante-mortem biomarker assessment. Several different underlying neuropathological diagnoses, including PART, appear to be associated with SNAP. However, SNAP has primarily been a research term and has only been minimally utilized in clinical practice [2, 9-11].

To better understand how PART is being or not being recognized in current clinical practice, we sought to identify the primary and contributing diagnoses in individuals who clinically presented with MCI or dementia and who met neuropathologic criteria for PART at autopsy. We also sought to compare these diagnoses for people with PART to those with AD neuropathology (ADNP) and to determine if individuals with PART less frequently received a clinical diagnosis of AD compared to those with ADNP.

\section{Methods}

\section{Data source and study sample}

Data were obtained from the National Alzheimer's Coordinating Center (NACC) Uniform Data Set (UDS) and Neuropathology Data Set. NACC is a data repository for over thirty past and present NIA-funded Alzheimer's Disease Centers (ADC) from across the United States. ADCs contribute data to the UDS, a standardized set of clinical assessments that are collected approximately annually from ADC clinical core participants. Written informed consents were obtained from participants at each ADC and approved by the ADC's Institutional Review Board (IRB). Standardized postmortem neuropathological evaluations are obtained from a subset of ADC participants that consent to autopsy. Research using the NACC database was approved by the University of Washington IRB. The UDS and Neuropathology Data Set data are described in detail elsewhere [12-15].

As of the December 2017 freeze, data from 36,769 participants were included in the UDS, of which, 4964 had neuropathology data. Hence, data had been collected between September 2005 and December 2017. Participants were included in this analysis if they had an autopsy within two years of their last UDS visit and had MCI or dementia at that visit. Participants were excluded if they were missing a primary clinical etiologic diagnosis, were missing data on CERAD neuritic plaques, or had any of the following comorbidities: neuropathological Lewy body disease (including amygdala-predominant), neuropathological prion disease, frontotemporal lobar degeneration (FTLD) neuropathology with tau inclusions, FTLD neuropathology with transactive response DNA-binding protein 43 (TDP-43), FTLD neuropathology not otherwise specified, or neuropathological amyotrophic lateral sclerosis (ALS). The sample included 1354 participants with either no neuritic plaques (i.e., PART) or moderate or frequent neuritic plaques (i.e., ADNP).

\section{Clinical diagnosis}

At each UDS visit, participants are assessed for cognitive status (i.e., normal cognition, MCI, or dementia). If symptomatic (MCI or dementia) for cognitive impairment, participants are assigned a presumptive etiologic diagnosis. In other words, the underlying pathologic cause (presumptive etiologic diagnosis) of the cognitive disorder is assigned and a determination is made whether the given diagnosis is a primary, contributing, or noncontributing cause of the observed impairment. Diagnosis by single clinician, formal consensus panel, or other diagnostic methods is dependent on the routine practice at each ADC; however, all ADCs follow standard guidelines for the clinical diagnostic criteria for a cognitive disorder. In this paper, clinical diagnosis refers to the presumptive etiologic diagnosis made at the most recent UDS visit prior to autopsy, and we examine the distribution of these clinical diagnoses by the cognitive status assigned at the same visit.

\section{Statistical analysis}

All statistical analyses were conducted using SAS version 9.4, and were stratified by cognitive status at last UDS visit (MCI or dementia). Independent $t$ tests and Chisquare analysis were used to assess whether subjects with PART and those with ADNP differed by demographics, apolipoprotein (APOE) \&4 carrier status, depression and AD clinical diagnosis at last UDS visit, history of TBI, amnestic vs. non-amnestic MCI (MCI participants only), and cerebrovascular neuropathological features. Exact Chi-square analysis was used if any cell sizes were less than 5. Multivariable logistic regression was used to assess if PART participants were significantly less likely to receive a primary or contributing clinical diagnosis of $\mathrm{AD}$ at their last visit. Models were adjusted for age at death, sex, education, and presence of $\geq 1$ APOE $\varepsilon 4$ allele. Generalized estimating equations were used to account for ADC clustering. 
Table 1 Demographics and clinical characteristics at last visit before death in symptomatic individuals with PART vs. ADNP, as of December 2017 data freeze

\begin{tabular}{|c|c|c|c|c|c|c|}
\hline \multirow[t]{2}{*}{ Characteristic $^{\mathrm{a}}$} & \multicolumn{2}{|c|}{ MCI diagnosis at last visit } & \multirow[b]{2}{*}{$p$-value $\mathrm{b}^{\mathrm{b}}$} & \multicolumn{2}{|c|}{ Dementia diagnosis at last visit } & \multirow[b]{2}{*}{$p$-value ${ }^{\mathrm{b}}$} \\
\hline & PART $(n=49)$ & $\operatorname{ADNP}(n=75)$ & & $\operatorname{PART}(n=112)$ & $\operatorname{ADNP}(n=1118)$ & \\
\hline Age at death, mean (SD) & $84.7(12.2)$ & $88.1(7.2)$ & 0.08 & $82.4(13.3)$ & $80.5(11.0)$ & 0.16 \\
\hline Male, $n(\%)$ & $28(57.1 \%)$ & $35(46.7 \%)$ & 0.25 & $65(58.0 \%)$ & $593(53.0 \%)$ & 0.31 \\
\hline Education (years), mean (SD) & $15.0(2.6)$ & $15.7(2.8)$ & 0.15 & $14.9(3.4)$ & $15.1(3.3)$ & 0.53 \\
\hline Non-white race, $n(\%)$ & $1(2.1 \%)$ & $4(5.4 \%)$ & 0.65 & $11(10.1 \%)$ & $73(6.6 \%)$ & 0.17 \\
\hline$\geq 1$ APOE e4 allele, $n(\%)$ & $7(15.2 \%)$ & $22(32.4 \%)$ & 0.04 & $15(14.9 \%)$ & $539(55.1 \%)$ & $<.0001$ \\
\hline Depression at last visit, $n(\%)$ & $30(61.2 \%)$ & $39(52.0 \%)$ & 0.31 & $65(58.0 \%)$ & $620(55.5 \%)$ & 0.60 \\
\hline History of TBI, $n(\%)$ & $10(20.4 \%)$ & $15(20.0 \%)$ & 0.96 & $22(19.6 \%)$ & $155(13.9 \%)$ & 0.10 \\
\hline \multicolumn{7}{|l|}{ MCI type, $n(\%)$} \\
\hline Amnestic & $33(67.4 \%)$ & $61(81.3 \%)$ & 0.08 & N/A & N/A & N/A \\
\hline Non-amnestic & $16(32.7 \%)$ & $14(18.7 \%)$ & & N/A & N/A & N/A \\
\hline Microinfarct(s) & $13(26.5 \%)$ & $17(22.7 \%)$ & 0.62 & $30(26.8 \%)$ & $199(17.8 \%)$ & 0.02 \\
\hline Hemorrhage(s)/microbleed(s) & $3(6.1 \%)$ & $6(8.5 \%)$ & 0.74 & $9(8.1 \%)$ & $68(6.1 \%)$ & 0.42 \\
\hline Infarct(s)/lacune(s) & $16(32.7 \%)$ & $19(25.7 \%)$ & 0.40 & $38(33.9 \%)$ & $225(20.2 \%)$ & 0.0008 \\
\hline Moderate/severe Arteriolosclerosis & $15(34.9 \%)$ & $29(45.3 \%)$ & 0.28 & $57(52.8 \%)$ & $430(43.3 \%)$ & 0.06 \\
\hline
\end{tabular}

${ }^{a}$ Missing data: [PART-MCI] APOE (3), race (1), arteriolosclerosis (6); [ADNP-MCI] education (1), APOE (7), race (1), hemorrhage/microbleed (4), infarct/lacune (1), arteriolosclerosis (11); [PART-dementia] education (3), APOE (11), race (3) hemorrhage/microbleed (1), arteriolosclerosis (4); [ADNP-dementia] education (9), APOE (140), race (6), microinfarct (2), hemorrhage/microbleed (11), infarct/lacune (6), arteriolosclerosis (124)

${ }^{\mathrm{b}}$ Used exact chi-square if any cell size $<5$

\section{Results}

Our final sample included a total of 1354 participants, with 161 individuals with PART (i.e., no neuritic plaques) and 1193 individuals with ADNP (i.e., moderate or frequent neuritic plaques). The PART and ADNP groups were stratified by cognitive status at their last UDS visit which resulted in 49 PART-MCI, 112 PART-dementia, 75 ADMCI, and 1118 AD-dementia. Our analyses excluded 155 PART and 86 ADNP participants who were diagnosed with normal cognition at their UDS visit prior to autopsy. Demographic and clinical characteristics, as well as cerebrovascular neuropathological features of the groups are described in Table 1. In those with MCI, ADNP participants were significantly more likely to be an $A P O E \varepsilon 4$ carrier than those with PART (ADNP: 32.4\%, PART: $15.2 \%$ PART, $p$ $=0.04$ ). A larger difference in APOE $\varepsilon 4$ carriers was seen between ADNP-dementia and PART-dementia participants, with a reported $55.1 \%$ of ADNP-dementia participants having at least one $A P O E \varepsilon 4$ allele, compared to $14.9 \%$ in those with PART-dementia $(p<0.0001)$. PART-dementia participants were more likely to have microinfarcts (ADNP: $17.8 \%$, PART: $26.8 \%, p=0.02)$ and infarcts or lacunes (ADNP: $20.9 \%$, PART: $33.9 \%, p=0.0008$ ) than those with ADNP-dementia.

Among PART-MCI, $57.1 \%$ were given a primary clinical diagnosis of $\mathrm{AD}$ (Table 2). Those who were not clinically diagnosed with $\mathrm{AD}$ were most commonly diagnosed with vascular brain injury (VBI; 8.2\%), ALS (6.1\%), and multiple system atrophy (MSA; 6.1\%). Similarly, in those with ADNP-MCI, AD was the most frequent primary clinical diagnosis (69.3\%), with VBI as the second most frequent primary clinical diagnosis (17.3\%).

Clinical presentation for those with ADNP-dementia appeared to be more consistent from other cognitive disorders, as $85.8 \%$ received a primary clinical diagnosis of $\mathrm{AD}$, compared to PART-dementia who were diagnosed with primary $\mathrm{AD}$ only $51.8 \%$ of the time (Table 2). This difference in the distribution of $\mathrm{AD}$ clinical diagnosis between ADNP-dementia and PART-dementia was statistically significant $(p<0.0001$; not shown). PART-dementia participants, who were not diagnosed with primary $A D$, often received diagnoses of VBI (17.0\%) or primary progressive aphasia/behavioral variant frontotemporal dementia (PPA/bvFTD; 12.5\%).

Seventeen participants with PART-dementia (15.2\%; Table 3), who were not diagnosed with AD as their primary clinical diagnosis, had AD reported as a contributing clinical diagnosis. VBI, depression, and systemic disease/ medical illness were recurrent contributing clinical diagnoses among all four groups, although with varying frequency between groups. Contributing clinical diagnoses of depression were more common in those with PART than ADNP, in both MCI and dementia groups. 
Table 2 Primary clinical diagnoses at last visit before death in symptomatic individuals with PART vs. ADNP, as of December 2017 data freeze

\begin{tabular}{|c|c|c|c|c|}
\hline \multirow[t]{2}{*}{ Primary clinical diagnosis ${ }^{\mathrm{a}, \mathrm{b}}$} & \multicolumn{2}{|c|}{ MCI diagnosis at last visit } & \multicolumn{2}{|c|}{ Dementia diagnosis at last visit } \\
\hline & PART $(n=49)$ & $\operatorname{ADNP}(n=75)$ & $\operatorname{PART}(n=112)$ & $\operatorname{ADNP}(n=1118)$ \\
\hline $\mathrm{AD}$ & $28(57.1 \%)$ & $52(69.3 \%)$ & $58(51.8 \%)$ & $959(85.8 \%)$ \\
\hline DLB & $2(4.1 \%)$ & $0(0.0 \%)$ & $1(0.9 \%)$ & $28(2.5 \%)$ \\
\hline Parkinson's disease & $1(2.0 \%)$ & $0(0.0 \%)$ & $0(0.0 \%)$ & $5(0.5 \%)$ \\
\hline PSP & $0(0.0 \%)$ & $0(0.0 \%)$ & $3(2.7 \%)$ & $0(0.0 \%)$ \\
\hline CBD & $0(0.0 \%)$ & $0(0.0 \%)$ & $3(2.7 \%)$ & $16(1.4 \%)$ \\
\hline PPA or bvFTD & $2(4.1 \%)$ & $0(0.0 \%)$ & $14(12.5 \%)$ & $62(5.6 \%)$ \\
\hline Vascular brain injury/dementia & $4(8.2 \%)$ & $13(17.3 \%)$ & $19(17.0 \%)$ & $25(2.2 \%)$ \\
\hline Prion & $0(0.0 \%)$ & $0(0.0 \%)$ & $1(0.9 \%)$ & $0(0.0 \%)$ \\
\hline Down syndrome & $0(0.0 \%)$ & $0(0.0 \%)$ & $0(0.0 \%)$ & $1(0.1 \%)$ \\
\hline Hydrocephalus & $0(0.0 \%)$ & $0(0.0 \%)$ & $0(0.0 \%)$ & $2(0.2 \%)$ \\
\hline CNS neoplasm & $0(0.0 \%)$ & $0(0.0 \%)$ & $2(1.8 \%)$ & $1(0.1 \%)$ \\
\hline Depression & $0(0.0 \%)$ & $2(2.7 \%)$ & $0(0.0 \%)$ & $0(0.0 \%)$ \\
\hline Other psychiatric disease & $1(2.0 \%)$ & $0(0.0 \%)$ & $0(0.0 \%)$ & $0(0.0 \%)$ \\
\hline Alcohol abuse & $1(2.0 \%)$ & $0(0.0 \%)$ & $0(0.0 \%)$ & $0(0.0 \%)$ \\
\hline Medication & $0(0.0 \%)$ & $1(1.3 \%)$ & $0(0.0 \%)$ & $0(0.0 \%)$ \\
\hline Medical/systemic illness & $2(4.1 \%)$ & $4(5.3 \%)$ & $1(0.9 \%)$ & $0(0.0 \%)$ \\
\hline ALS & $3(6.1 \%)$ & $1(1.3 \%)$ & $2(1.8 \%)$ & $1(0.1 \%)$ \\
\hline MSA & $3(6.1 \%)$ & $0(0.0 \%)$ & $0(0.0 \%)$ & $0(0.0 \%)$ \\
\hline PCA & $0(0.0 \%)$ & $0(0.0 \%)$ & $0(0.0 \%)$ & $2(0.2 \%)$ \\
\hline Multiple sclerosis & $0(0.0 \%)$ & $0(0.0 \%)$ & $1(0.9 \%)$ & $0(0.0 \%)$ \\
\hline Probable CTE & $0(0.0 \%)$ & $0(0.0 \%)$ & $0(0.0 \%)$ & $1(0.1 \%)$ \\
\hline Unknown etiologic diagnosis & $1(2.0 \%)$ & $0(0.0 \%)$ & $5(4.5 \%)$ & $12(1.1 \%)$ \\
\hline Other misc. write-in & $1(2.0 \%)$ & $2(2.7 \%)$ & $2(1.8 \%)$ & $3(0.3 \%)$ \\
\hline
\end{tabular}

${ }^{\mathrm{a}}$ Each participant has only one primary diagnosis

${ }^{\mathrm{b}} A D$ Alzheimer's disease, $D L B$ dementia with Lewy bodies, $P S P$ progressive supranuclear palsy, $C D B$ corticobasal degeneration, $P P A$ primary progressive aphasia, $b v F T D$ behavioral variant frontotemporal dementia, $C N S$ central nervous system, $A L S$ amyotrophic lateral sclerosis, $M S A$ multiple system atrophy, $P C A$ posterior cortical atrophy, $C T E$ chronic traumatic encephalopathy
In the logistic regression analyses examining the odds of a clinical diagnosis of AD among those with PART compared to ADNP, reports of primary or contributing clinical diagnosis of AD were combined (Tables 4 and 5). Unadjusted models found that participants with primary/contributing clinical AD-MCI were often older at death than those who were not diagnosed with AD (OR: 1.07; 95\% CI: 10.1-1.13). Older death age (OR: 1.05 ; 95\% CI 1.04-1.06) and presence of an $A P O E \& 4$ allele (OR: 1.66; 95\% CI: 1.13-2.44) was observed more in those who were diagnosed with a primary/contributing clinical AD-dementia than in those who were not. Male sex (OR: 0.65; 95\% CI $0.50-0.85$ ) was less frequent among those with a clinical primary/contributing AD-dementia diagnosis.

Primary/contributing clinical diagnoses of $\mathrm{AD}$ was less likely in participants with PART neuropathology vs. ADNP participants with dementia (OR: 0.19; 95\% CI: 0.12-0.31; Table 4). Even after adjusting for age at death, sex, education, and $A P O E \& 4$ allele carrier status, this relationship persisted (OR: 0.22 , 95\% CI: 0.13-0.38; Table 5).

\section{Discussion}

In this study, we report the primary and contributing diagnoses in individuals who had ante-mortem clinical presentation of MCI or dementia and who met neuropathological criteria for PART at eventual autopsy. We found that people who had PART were most commonly categorized as $\mathrm{AD}$, especially during the MCI stage. The percent of people diagnosed as having $\mathrm{AD}$ was less in the dementia stage, but even then, the majority of people with PART were still being diagnosed as having AD. Nonetheless, almost half of people with PART and dementia were diagnosed as something other than $\mathrm{AD}$, particularly primary progressive aphasia/behavioral variant frontotemporal dementia (PPA/bvFTD), or vascular brain injury.

Given that $\mathrm{AD}$ is highly prevalent, and that there are currently no ante-mortem diagnostic criteria for PART, it is not surprising that most individuals who eventually were determined to have PART were diagnosed as having AD while alive. Moreover, given that mixed pathology is 
Table 3 Contributing clinical diagnoses at last visit before death in symptomatic individuals with PART vs. ADNP, as of December 2017 data freeze

\begin{tabular}{|c|c|c|c|c|}
\hline \multirow{2}{*}{$\begin{array}{l}\text { Contributing } \\
\text { clinical } \\
\text { diagnosis }^{\mathrm{a}, \mathrm{b}}\end{array}$} & \multicolumn{2}{|c|}{$\begin{array}{l}\text { MCI diagnosis at last } \\
\text { visit }\end{array}$} & \multicolumn{2}{|c|}{$\begin{array}{l}\text { Dementia diagnosis at } \\
\text { last visit }\end{array}$} \\
\hline & $\begin{array}{l}\text { PART } \\
(n=49)\end{array}$ & $\begin{array}{l}\text { ADNP } \\
(n=75)\end{array}$ & $\begin{array}{l}\text { PART } \\
(n=112)\end{array}$ & $\begin{array}{l}\text { ADNP } \\
(n=1118)\end{array}$ \\
\hline $\mathrm{AD}$ & $1(2.0 \%)$ & $5(6.7 \%)$ & $17(15.2 \%)$ & $57(5.1 \%)$ \\
\hline DLB & $2(4.1 \%)$ & $0(0.0 \%)$ & $1(0.9 \%)$ & $35(3.1 \%)$ \\
\hline $\begin{array}{l}\text { Parkinson's } \\
\text { disease }\end{array}$ & $0(0.0 \%)$ & $0(0.0 \%)$ & $1(0.9 \%)$ & $9(0.8 \%)$ \\
\hline CBD & $0(0.0 \%)$ & $0(0.0 \%)$ & $1(0.9 \%)$ & $5(0.5 \%)$ \\
\hline PSP & $0(0.0 \%)$ & $0(0.0 \%)$ & $1(0.9$ & $1(0.1 \%)$ \\
\hline bvFTD or PPA & $1(2.0 \%)$ & $2(2.7 \%)$ & $2(1.8 \%)$ & $23(2.1 \%)$ \\
\hline FTLD NOS & $0(0.0 \%)$ & $0(0.0 \%)$ & $0(0.0 \%)$ & $1(0.1 \%)$ \\
\hline $\begin{array}{l}\text { Vascular brain } \\
\text { injury/ } \\
\text { dementia }\end{array}$ & $4(8.2 \%)$ & $6(8.0 \%)$ & $18(16.1 \%)$ & $111(9.9 \%)$ \\
\hline Prion disease & $0(0.0 \%)$ & $0(0.0 \%)$ & $0(0.0 \%)$ & $1(0.1 \%)$ \\
\hline Hydrocephalus & ) & $0(0.0 \%)$ & 1( & $6(0.5 \%)$ \\
\hline CNS neoplasm & $1(2.0 \%)$ & 0 & $0(0.0$ & $2(0.2 \%)$ \\
\hline Depression & $15(30.6 \%)$ & $12(16.0 \%)$ & $24(21.4 \%)$ & $87(7.8 \%)$ \\
\hline Schizophrenia & $0(0.0 \%)$ & $0(0.0 \%)$ & $0(0.0 \%)$ & $1(0.1 \%)$ \\
\hline Anxiety & $0(0.0 \%)$ & $0(0.0 \%)$ & $1(0.9 \%)$ & $3(0.3 \%)$ \\
\hline $\begin{array}{l}\text { Other } \\
\text { psychiatric } \\
\text { disease }\end{array}$ & $1(2.0 \%)$ & $0(0.0 \%)$ & $0(0.0 \%)$ & $5(0.5 \%)$ \\
\hline Alcohol abuse & $0(0.0 \%)$ & $0(0$ & 1( & $4(0.4 \%)$ \\
\hline $\begin{array}{l}\text { Systemic } \\
\text { disease/ } \\
\text { medical illness }\end{array}$ & $6(12.2 \%)$ & $2(2.7 \%)$ & $9(8.0 \%)$ & $22(2.0 \%)$ \\
\hline Medications & $0(0.0 \%)$ & $2(2.7 \%)$ & $4(3.6 \%)$ & $10(0.9 \%)$ \\
\hline $\begin{array}{l}\text { Essential } \\
\text { tremor }\end{array}$ & $1(2.0 \%)$ & $0(0.0 \%)$ & $0(0.0 \%)$ & $0(0.0 \%)$ \\
\hline TBI & $1(2.0 \%)$ & $0(0.0 \%)$ & $2(1.8 \%)$ & $13(1.2 \%)$ \\
\hline
\end{tabular}

${ }^{a}$ Each participant can have none, one, or more contributing diagnoses ${ }^{\text {b}} A D$ Alzheimer's disease, $D L B$ dementia with Lewy bodies, $P S P$ progressive supranuclear palsy, $C D B$ corticobasal degeneration, $P P A$ primary progressive aphasia, $b v F T D$ behavioral variant frontotemporal dementia, FTLD NOS frontotemporal lobar dementia not otherwise specified, $C N S$ central nervous system, TBI traumatic brain injury

common in the elderly [16], and that PART is relatively less symptomatic than $\mathrm{AD}$, other pathologies may indeed be playing a role in the production of symptoms of people who had underlying PART neuropathology. For example, the $17 \%$ of people with PART and dementia who were diagnosed as having vascular brain injury may have had either had the vascular insult as a contributor to or cause of their cognitive impairment, with PART itself playing a lesser or non-existent role. It is also notable that the assignment of the diagnoses of AD was similar between PART and ADNP for participants with MCI. However, in those with dementia, the distribution diverged, with $86 \%$ of persons with
Table 4 Unadjusted association between PART vs. AD neuropathology and clinical diagnosis of $\mathrm{AD}$ (primary or contributing), as of December 2017 data freeze

\begin{tabular}{|c|c|c|c|c|}
\hline \multirow[t]{2}{*}{ Characteristic } & \multicolumn{2}{|c|}{$\begin{array}{l}\text { MCI diagnosis } \\
\text { at last visit }\end{array}$} & \multicolumn{2}{|c|}{$\begin{array}{l}\text { Dementia } \\
\text { diagnosis at last } \\
\text { visit }\end{array}$} \\
\hline & $\overline{\mathrm{OR}}$ & $95 \% \mathrm{CI}$ & $\overline{\mathrm{OR}}$ & $95 \% \mathrm{CI}$ \\
\hline Age at death (years) & 1.07 & 1.01-1.13 & 1.05 & $1.04-1.06$ \\
\hline Male & 0.72 & $0.33-1.58$ & 0.65 & $0.50-0.85$ \\
\hline Education (years) & 1.00 & $0.87-1.14$ & 0.97 & $0.92-1.02$ \\
\hline$\geq 1$ APOE e4 allele & 1.01 & $0.41-2.48$ & 1.66 & $1.13-2.44$ \\
\hline PART (vs. ADNP) & 0.39 & $0.15-1.03$ & 0.19 & $0.12-0.31$ \\
\hline Microinfarct & 0.56 & $0.29-1.08$ & 0.82 & $0.50-1.34$ \\
\hline Hemorrhage/microbleed & 1.23 & $0.31-4.93$ & 1.04 & $0.45-2.43$ \\
\hline $\begin{array}{l}\text { Moderate/severe } \\
\text { arteriolosclerosis }\end{array}$ & 1.10 & $0.42-2.92$ & 1.11 & $0.72-1.70$ \\
\hline Infarct/lacune & 0.66 & $0.29-1.51$ & 0.73 & $0.48-1.12$ \\
\hline
\end{tabular}

${ }^{a}$ Bold values indicate significant findings $(p<0.05)$

Table 5 Adjusted association between PART vs. AD neuropathology and clinical diagnosis of $\mathrm{AD}$ (primary or contributing), as of December 2017 data freeze

\begin{tabular}{llllll}
\hline Characteristic & \multicolumn{2}{l}{$\begin{array}{l}\text { MCI diagnosis at } \\
\text { last visit }\end{array}$} & & \multicolumn{2}{l}{$\begin{array}{l}\text { Dementia diagnosis } \\
\text { at last visit }\end{array}$} \\
\cline { 2 - 3 } & OR & $95 \%$ CI & & OR & $95 \%$ CI \\
\hline Age at death (years) & $\mathbf{1 . 0 6}$ & $\mathbf{1 . 0 1 - 1 . 1 1}$ & & $\mathbf{1 . 0 6}$ & $\mathbf{1 . 0 4 - 1 . 0 7}$ \\
Male & 0.90 & $0.36-2.24$ & & 0.82 & $0.59-1.14$ \\
Education (years) & 0.99 & $0.82-1.20$ & & 0.96 & $0.91-1.02$ \\
$\geq 1$ APOE e4 allele & 1.00 & $0.39-2.58$ & & 1.43 & $0.92-2.22$ \\
PART (vs. ADNP) & 0.53 & $0.25-1.12$ & $\mathbf{0 . 2 2}$ & $\mathbf{0 . 1 3 - 0 . 3 8}$
\end{tabular}

${ }^{\mathrm{a} B o l d}$ values indicate significant findings $(p<0.05)$

ADNP being assigned a diagnosis of $\mathrm{AD}$, in comparison to only $52 \%$ of participants with PART. Thus, it appears that clinicians are perceiving a notable difference in how dementia symptoms manifest clinically in people with PART neuropathology in comparison to people with ADNP.

In the literature, several older studies reported on symptoms and diagnoses of people with senile dementia of the tangle type or with neurofibrillary tangle predominant form of senile dementia, both of which are older terms for what has now been termed PART $[2,5,6]$. One of these earlier studies reported on 10 cases, all of whom had been diagnosed with possible or probable AD during life [6]. In 2003, one study suggested criteria for clinical diagnosis of "senile dementia of the neurofibrillary tangle type (tangleonly dementia)". These criteria included later age at onset, isolated memory disturbance at onset, slower progression, and CT or MRI findings of mild to moderate diffuse cortical and hippocampal atrophy with enlarged inferior horns of the 
lateral ventricles. However, the author went on to caution that development of reliable diagnostic tests would be necessary to reliably differentiate this entity from $\mathrm{AD}$ or other neurodegenerative dementias [17]. The current study adds to the literature by having a larger sample size (161 people with PART), by applying the latest consensus definition of PART [2], and by undertaking a comparison with people with ADNP. The current study also shows new findings of potential non-amnestic symptoms, as indicated by the assignment of diagnoses such as PPA and bvFTD.

In recent years, the category of suspected non-Alzheimer disease pathophysiology (SNAP) has been increasingly recognized in living persons. SNAP denotes the finding of neurodegeneration in the absence of amyloid deposits in the brain. It may be present in persons independent of the presence, absence, or severity of cognitive impairment. The determination of SNAP is based primarily on biomarkers, including fluid biomarkers and neuroimagining. SNAP appears to overlap with PART. Although autopsy correlations have been limited thus far, several underlying neuropathological diagnoses appear to be associated with SNAP, including PART, hippocampal sclerosis, argyrophilic grain disease, cerebrovascular disorders, mixed pathologies, and TDP-43, among others. The relative proportions of each of these neuropathologic diagnoses in people with SNAP have not been well worked out [2, 9-11]. There is also some evidence that the overlap between SNAP and PART may be fairly minimal, at least in people who are clinically normal. For example, one recent study looked at clinically normal people with SNAP and found minimal evidence of elevated tau on PET imaging [18]. Moreover, SNAP has primarily been a research term and has only been minimally utilized in clinical practice [2, 9-11].

The term SNAP is not listed as a choice for diagnosis in the UDS. However, clinicians assessing the presumptive etiology of the cognitive disorder have an option to write in a diagnosis that is not in the standardized list. In the NACC database, as of the December 2017 data freeze, there have been only twenty-three participants who had SNAP listed as a diagnosis. All of these were alive at that time and hence none were included in the current study. With increasing understanding of both PART and SNAP, it is possible that use of this diagnosis might grow in the future, especially if it were to be offered as a choice for diagnosis in the UDS.

This study had important limitations. First, the NACC database is derived from convenience samples at each ADC. Participants tend to be more highly educated and with a greater proportion of people of white race than the population at large. These factors limit our ability to draw inferences to the general population. However, these demographic factors were not differentially distributed between the two groups (PART and ADNP) analyzed in the study. Second, we utilized the presence of neuritic plaques (i.e., CERAD score) as an inclusion criterion for ADNP. Diffuse plaques (i.e., Thal phase) have recently been incorporated into the criteria for neuropathologic diagnosis of ADNP $[19,20]$, and adopted as part of the NACC neuropathology evaluation in NP version 10 , implemented in 2014. Therefore, Thal phase is not available for most participants in the NACC database. Because of this, some participants classified as PART in this study might have been classified as ADNP if Thal phase had been used in addition to the CERAD neuritic plaque score. However, it also appears that diffuse plaques contribute less to antemortem cognitive change than neuritic plaques [21]. Hence, the effect of such classification on study findings is likely small. Lastly, there are limited data available in the NACC database about whether ante-mortem biomarker assays were run to determine amyloid burden, or if available test results were considered when making the primary clinical diagnosis. While ante-mortem criteria for diagnosis of PART are not established, if participants were tested for ante-mortem biomarkers, the results may have altered the clinical diagnosis. Despite these limitations, this study has several notable strengths. These include the fact that data were collected from multiple centers in widely separated geographic areas (increasing the generalizability of the data) using standardized methods for gathering clinical data and classifying cognitive status and for performing and reporting autopsy findings.

In conclusion, this study suggests that clinicians may recognize the clinical distinction between PART and ADNP, diagnosing AD less frequently in those with PART, especially for patients with dementia. Nonetheless, clinical AD was diagnosed greater than $50 \%$ of the time in PART participants with MCI or dementia. As PART is being increasingly recognized as a neuropathological entity that is distinct from ADNP and that has its own, usually less severe, cognitive outcomes, ante-mortem criteria for diagnosis of PART need to be established.

Acknowledgements The NACC database is funded by NIA/NIH Grant U01 AG016976. NACC data are contributed by the NIA-funded ADCs: P30 AG019610 (PI Eric Reiman, MD), P30 AG013846 (PI Neil Kowall, MD), P50 AG008702 (PI Scott Small, MD), P50 AG025688 (PI Allan Levey, MD, PhD), P50 AG047266 (PI Todd Golde, MD, PhD), P30 AG010133 (PI Andrew Saykin, PsyD), P50 AG005146 (PI Marilyn Albert, PhD), P50 AG005134 (PI Bradley Hyman, MD, PhD), P50 AG016574 (PI Ronald Petersen, MD, PhD), P50 AG005138 (PI Mary Sano, PhD), P30 AG008051 (PI Thomas Wisniewski, MD), P30 AG013854 (PI M. Marsel Mesulam, MD), P30 AG008017 (PI Jeffrey Kaye, MD), P30 AG010161 (PI David Bennett, MD), P50 AG047366 (PI Victor Henderson, MD, MS), P30 AG010129 (PI Charles DeCarli, MD), P50 AG016573 (PI Frank LaFerla, PhD), P50 AG005131 (PI James Brewer, MD, PhD), P50 AG023501 (PI Bruce Miller, MD), P30 AG035982 (PI Russell Swerdlow, MD), P30 AG028383 (PI Linda Van Eldik, PhD), P30 AG053760 (PI Henry Paulson, MD, PhD), P30 AG010124 (PI John Trojanowski, MD, PhD), P50 AG005133 (PI Oscar Lopez, MD), P50 
AG005142 (PI Helena Chui, MD), P30 AG012300 (PI Roger Rosenberg, MD), P30 AG049638 (PI Suzanne Craft, PhD), P50 AG005136 (PI Thomas Grabowski, MD), P50 AG033514 (PI Sanjay Asthana, MD, FRCP), P50 AG005681 (PI John Morris, MD), P50 AG047270 (PI Stephen Strittmatter, MD, PhD).

\section{Compliance with ethical standards}

Conflict of interest The authors declare that they have no conflict of interest.

Publisher's note: Springer Nature remains neutral with regard to jurisdictional claims in published maps and institutional affiliations.

\section{References}

1. Besser LM, Crary JF, Mock C, et al. Comparison of symptomatic and asymptomatic persons with primary age-related tauopathy. Neurology. 2017;89:1707-15.

2. Crary JF, Trojanowski JQ, Schneider JA, et al. Primary agerelated tauopathy (PART): a common pathology associated with human aging. Acta Neuropathol. 2014;128:755-66.

3. Crary JF. Primary age-related tauopathy and the amyloid cascade hypothesis: the exception that proves the rule? J Neurol Neuromedicine. 2016;1:53-7.

4. Kryscio RJ, Abner EL, Nelson PT, et al. The effect of vascular neuropathology on late-life cognition: results from the SMART project. J Prev Alzheimers Dis. 2016;3:85-91.

5. Ikeda K, Akiyama H, Arai T, et al. Clinical aspects of 'senile dementia of the tangle type'-- a subset of dementia in the senium separable from late-onset Alzheimer's disease. Dement Geriatr Cogn Disord. 1999;10:6-11.

6. Bancher C, Jellinger KA. Neurofibrillary tangle predominant form of senile dementia of Alzheimer type: a rare subtype in very old subjects. Acta Neuropathol. 1994;88:565-70.

7. Ranginwala NA, Hynan LS, Weiner MF, et al. Clinical criteria for the diagnosis of Alzheimer disease: still good after all these years. Am J Geriatr Psychiatry. 2008;16:384-8.

8. Beach TG, Monsell SE, Phillips LE, et al. Accuracy of the clinical diagnosis of Alzheimer disease at national institute on aging Alzheimer disease centers, 2005-2010. Neuropathol Exp Neurol. 2012;71:266-73.
9. Jack CR Jr., Knopman DS, Chetelat G, et al. Suspected nonAlzheimer disease pathophysiology--concept and controversy. Nat Rev Neurol. 2016;12:117-24.

10. Wisse LEM, Butala N, Das SR, et al. Alzheimer's disease neuroimaging I. suspected non-AD pathology in mild cognitive impairment. Neurobiol Aging. 2015;36:3152-62.

11. Yamada M. Suspected Non-Alzheimer's Disease Pathophysiology (SNAP) and Its Pathological Backgrounds in the Diagnosis of Preclinical and Clinical Alzheimer's Disease. Brain Nerve. 2018;70:59-71.

12. Morris JC, Weintraub S, Chui HC, et al. The Uniform Data Set (UDS): clinical and cognitive variables and descriptive data from Alzheimer Disease Centers. Alzheimer Dis Assoc Disord. 2006;20:210-6.

13. Beekly DL, Ramos EM, Lee WW, et al. The National Alzheimer's Coordinating Center (NACC) database: the Uniform Data Set. Alzheimer Dis Assoc Disord. 2007;21:249-58.

14. Beekly DL, Ramos EM, van Belle G, et al. The National Alzheimer's Coordinating Center (NACC) database: an Alzheimer disease database. Alzheimer Dis Assoc Disord. 2004;18:270-7.

15. Besser LM, Kukull WA, Teylan MA, et al. The Revised National Alzheimer's Coordinating Center's Neuropathology FormAvailable Data and New Analyses. J Neuropathol Exp Neurol. 2018;77:717-26.

16. Schneider JA, Arvanitakis Z, Bang W, et al. Mixed brain pathologies account for most dementia cases in communitydwelling older persons. Neurology. 2007;69:2197-204.

17. Yamada M. Senile dementia of the neurofibrillary tangle type (tangle-only dementia): neuropathological criteria and clinical guidelines for diagnosis. Neuropathology. 2003;23:311-7.

18. Mormino EC, Papp KV, Rentz DM, et al. Heterogeneity in suspected non-alzheimer disease pathophysiology among clinically normal older individuals. JAMA Neurol. 2016;73:1185-91.

19. Thal DR, Rub U, Orantes M, et al. Phases of A beta-deposition in the human brain and its relevance for the development of AD. Neurology. 2002;58:1791-1800.

20. Sperling RA, Aisen PS, Beckett LA, et al. Toward defining the preclinical stages of Alzheimer's disease: recommendations from the National Institute on Aging-Alzheimer's Association workgroups on diagnostic guidelines for Alzheimer's disease. Alzheimers Dement. 2011;7:280-92.

21. Serrano-Pozo A, Qian J, Muzikansky A, et al. Thal amyloid stages do not significantly impact the correlation between neuropathological change and cognition in the Alzheimer disease continuum. J Neuropathol Exp Neurol. 2016;75:516-26. 